\title{
HYPERCENTRAL RESIDUALS OF A JOIN OF SUBNORMAL SUBGROUPS
}

\author{
JENNIFER WHITEHEAD
}

\begin{abstract}
It is proved that the hypercentral residual of a group of finite rank, generated by two subnormal subgroups, is the join of the hypercentral residuals of the generating subnormal subgroups. The theorem is subject to the condition that, when factored by their hypercentral residuals, these groups are hypercentral.
\end{abstract}

1. Introduction. Let $G$ be a group, and $\mathfrak{X}$ a class of groups. The $\mathfrak{X}$-residual of $G$ is defined to be the intersection of all normal subgroups of $G$ whose factor groups are $\mathfrak{X}$-groups. If $\mathfrak{X}$ is the class of hypercentral groups, we shall denote the hypercentral residual of $G$ by $G^{*}$.

Recall that a group has finite rank $r$ if every finitely generated subgroup of $G$ can be generated by $r$ elements, and $r$ is the smallest integer with this property. Since locally nilpotent groups of finite rank are hypercentral [6, Part 2, p. 38], for a group $G$ of finite rank, the hypercentral residual $G^{*}$ and the locally nilpotent residual coincide. We now state:

THEOREM. Let $G$ be a group generated by two subnormal subgroups $H$ and $K$ of finite rank. If $H / H^{*}$ and $K / K^{*}$ are hypercentral then $G^{*}=\left\langle H^{*}, K^{*}\right\rangle=H^{*} K^{*}$ and $G / G^{*}$ is hypercentral of finite rank.

For locally nilpotent residuals, this was established in [9] for the class of locally finite groups. Wielandt [10] originally obtained these results for nilpotent residuals of groups having a composition series, and Stonehewer [8] obtained a generalization to groups of finite rank. Stronger results concerning the lower central series of a group have recently been obtained in [4].

2. Notation and preliminary results. If $\mathfrak{X}$ and $\mathfrak{Y}$ are classes of groups, then $\mathfrak{X} \mathfrak{Y}$ denotes the class of groups with a normal subgroup $N$ such that $N \in \mathfrak{X}$ and $G / N \in \mathfrak{Y}$. Then $\mathfrak{X}^{n}$ denotes the class of groups with an $\mathfrak{X}$-series of length $n$. We denote the class of locally nilpotent groups by $L \mathfrak{R}$, and the class of abelian groups by $\mathfrak{A}$. If $H$ is a subgroup of $G$, we define $H_{0}=G$ and inductively $H_{i+1}=H^{H_{i}}$ for $i \geqslant 0 . H_{i}$ is called the $i$ th normal closure of $H$ in $G$. If $H$ is subnormal in $G$ we write $H \operatorname{sn} G$; and if the index of subnormality is at most $n$, then we write $H \triangleleft^{n} G$. Hence $H \triangleleft^{n} G$ if and only if $H=H_{n}$. For a group $G$ we write $\zeta_{r}(G)$ to denote the $r$ th term of the upper central series of $G$.

Received by the editors May 8, 1980.

AMS (MOS) subject classifications (1970). Primary 20F30, $20 \mathrm{E} 20$.

Key words and phrases. Subnormal subgroup, hypercentral residual, finite rank.

(C)1982 American Mathematical Society 0002-9939/81/0000-1098/\$02.00 
The following result on the hypercentral residual of a group $G$ is clear.

LEMMA 2.1. Suppose that $\theta$ is a homomorphism of a group $G$ and that $G / G^{*}$ is hypercentral. Then $(G \theta)^{*}=\left(G^{*}\right) \theta$ and $G \theta /(G \theta)^{*}$ is hypercentral.

Henceforth, let $G, H, K$ be as in the theorem. Let $B=\left\langle H^{*}, K^{*}\right\rangle$ and $A=G^{*}$; then $B \leqslant A$.

LEMMA 2.2. With the hypotheses as in the theorem, if $B A^{\prime}=A$ then $A=B$.

Proof. Since the class of groups of finite rank forms a subnormal coalition class [1], $B$ is subnormal in $G$ and $G$ has finite rank.

Let $L=\langle H, B\rangle$, then $L \operatorname{sn} G$ and we may suppose that

$$
L=L_{r} \triangleleft L_{r-1} \triangleleft \cdots \triangleleft L_{1} \triangleleft G
$$

is the normal closure series of $L$ in $G$. We show by induction that $A \leqslant L_{i}, 1 \leqslant i \leqslant r$.

Now $G / L_{1} \cong L_{1} K / L_{1} \cong K / K \cap L_{1} \in L \Re$. By definition of $A$ we have $A \leqslant L_{1}$. Suppose by induction that $A \leqslant L_{i-1}$. Let $M=A \cap L_{i}$ and $N=M^{K}$; then since $L_{i}$ centralizes $A / M, L_{i}$ normalizes $N$ and centralizes $A / N$. Thus $N \triangleleft\left\langle L_{i}, K\right\rangle=G$ and $L_{i}^{G}=L_{1}$ centralizes $A / N$. Since $A \leqslant L_{1}$, it follows that $A / N$ is abelian and since $B \leqslant N$ we have $N=A$ by hypothesis. Thus $M \triangleleft A \triangleleft\langle M, K\rangle$ and $K^{*} \leqslant M \cap K$. Applying [7, Lemma 1] there exists a positive integer $s$ such that $A / M \in(L \mathfrak{R}) \mathfrak{A}^{s}$. Since a perfect hypercentral group is trivial by Grün's lemma (see [3, Vol. II, p. 227]), and $B \leqslant M$, we obtain $M=A$ and $A \leqslant L_{i}$, whence $A \leqslant L$.

Let $B=B_{n} \triangleleft B_{n-1} \triangleleft \cdots \triangleleft B_{1} \triangleleft L$ be the normal closure series of $B$ in $L$. Then $B_{1}=B^{L} \leqslant A$ and $L / B_{1} \in L \Re$ whence $A / B_{1} \in L \Re$. By hypothesis $A=B_{1}$. Again by [7], there exists a positive integer $t$ such that $A / B_{2} \in L \mathfrak{R} \mathfrak{U}^{t}$ and so $B_{2}=A=B_{1}$. Since $B \operatorname{sn} A$ we obtain $B=A$ as required.

3. Proof of the theorem. As in Lemma 2.2, $B$ sn $G$ and $G$ has finite rank. Then $G / A$ is generated by the subnormal locally nilpotent subgroups $H A / A, K A / A$ and hence is locally nilpotent [6, Part 2, p. 57]. Since $G$ has finite $\operatorname{rank}, G / A$ has finite rank and hence is hypercentral. It is now sufficient to show that $A=B$ for by [9, Theorem C] this implies $A=B=H^{*} K^{*}$.

By Lemma $2.2 A^{\prime} B<A$ and by Lemma 2.1 we may factor $G$ by $A^{\prime}$ and assume $A$ is abelian. Let $C=C_{G}(A)$, the centralizer of $A$ in $G$; then $C$ is hypercentral since $G / A$ is hypercentral. By [9] $(C H)^{*}=H^{*}$ and $(C K)^{*}=K^{*}$ whence we may assume that $C \leqslant H \cap K$. Let $G_{0}$ be the split extension of $A$ by $G / C$ generated by the split extensions of $A$ by $H / C$ and $A$ by $K / C$, respectively named $H_{0}$ and $K_{0}$. Then $H_{0}$ and $K_{0}$ are subnormal subgroups of $G_{0}$ and $G_{0}^{*}=A, H_{0}^{*}=H^{*}$ and $K_{0}^{*}=K^{*}$. It is therefore sufficient to prove the theorem for $G_{0}, H_{0}$ and $K_{0}$ in place of $G, H$ and $K$.

Following the proof of Theorem $\mathrm{B}$ of [8], there exists a subgroup $X, B \leqslant X<A$, such that $A / X$ is a $p$-group for some prime $p$. Without loss of generality we may assume Core ${ }_{G} X=1$. Let $y$ be an element of prime order $q$ in $A$ where $q$ is a prime, $q \neq p$. Then there exists $g \in G$ such that $y \notin X^{g}$. Since $A / X^{g} \cong A / X$, a $p$-group, 
this contradiction implies that the torsion subgroup $T$ of $A$ is a $p$-group. Thus there exists a finite series of normal subgroups in $G$,

$$
1=A_{0} \triangleleft A_{1} \triangleleft \cdots \triangleleft A_{s}=A,
$$

where each factor is either a torsion-free abelian group of finite rank, a finite elementary abelian $p$-group or a direct product of finitely many groups of type $p^{\infty}$. Then by [6, Part 1, p. 78] $G / A$ is soluble and by Mal'cev's structure theorem for soluble linear groups $G$ has a normal subgroup $N$, of finite index, containing $A$ such that $\left[A,{ }_{r} N^{\prime}\right]=1$ for some integer $r$. Since $G / A$ is hypercentral, this implies that $N^{\prime}$ is hypercentral. By [9] $\left(N^{\prime} H\right)^{*}=H^{*}$ and $\left(N^{\prime} K\right)^{*}=K^{*}$ and we may assume that $N^{\prime} \leqslant H \cap K$. Since $H \cap K \leqslant N_{G}(B)$, the above argument and the fact that $B$ is core-free implies that $B\left[A, N^{\prime}\right]<A$. Therefore we may factor by $\left[A, N^{\prime}\right]$ according to Lemma 2.1, and assume $\left[A, N^{\prime}\right]=1$. Since $A=C_{G}(A)$, we obtain $N^{\prime} \leqslant A$ and $G / A$ is abelian-by-finite. A similar argument enables us to assume that every normal nilpotent subgroup of $G$ is contained in $A$. We therefore conclude that $A=\mathbf{F}(G)$ where $\mathrm{F}(G)$ denotes the Fitting subgroup of $G$.

We recall that the torsion subgroup $T$ of $A$ is Črnikov and that $G$ splits over $A$. Thus there exists a hypercentral subgroup $S$ of $G$ such that $G=S A$ and $S \cap A=1$. Since $A \leqslant H \cap K$ it follows that $H=A(H \cap S)$ and $K=A(K \cap S)$. Since $S_{1}=$ $\langle H \cap S, K \cap S\rangle \leqslant S$ and $S_{1}$ complements $A$ in $G$ we obtain $S=S_{1}$.

We now consider two cases.

Case 1. $B T=A$. Let $Z / A=\zeta_{1}(G / A)$; then $Z \leqslant N_{G}(H) \cap H_{G}(K)$. Since $H^{*}$ and $K^{*}$ are characteristic in $H$ and $K$ respectively $Z \leqslant N_{G}\left(H^{*}\right) \cap N_{G}\left(K^{*}\right)$. Therefore $Z$ normalizes $B$. If $C_{G}(T) \neq A$ then $C_{G}(T) \cap Z \neq A$ (see [6, Part 1, p. 48]). Let $z \in\left(Z \cap C_{G}(T)\right) \backslash A$, then $[A, z]=[B T, z]=[B, z] \leqslant B$. Since $[A, z] \triangleleft G$ and $B$ is core-free we obtain $z \in A$. This contradiction implies that $C_{G}(T)=A$. Since $A=\mathbf{F}(G)$ and $G / \mathbf{F}(G)$ is residually finite [5, Theorem B], it follows that $G / A$ contains no nontrivial radicable subgroups. Thus if $P / A$ is the torsion subgroup of $N / A, P / A$ is Černikov since $T$ is Černikov [6, Part 1, Theorem 3.29] and by the above, $P / A$ is finite. Let $|P / A|=n$; then $L / A=(N / A)^{n}$ is a torsion-free characteristic subgroup of $N / A$ and $|G: L|<\infty$. It follows from [6, Part 2, Lemma 6.37] that $L / A \leqslant Z / A$. Therefore $L \leqslant N_{G}(B)$ and $\left|G: N_{G}(B)\right|<\infty$. Since $A / B$ is a $p$-group and $B$ is core-free, $A$ is a $p$-group and $A \in \operatorname{Min}$. If $A$ is finite then $G$ is finite and the theorem is true by [10]. Thus we assume $A$ is infinite.

Let $\left\{a_{1}, a_{2}, \ldots, a_{t}\right\}$ be any finite set of elements of $A$. By the structure of groups satisfying the minimal condition, for each $i$ there are only finitely many elements of $A$ with order equal to that of $a_{i}$. Therefore $\left|G: C_{i}\left(a_{i}\right)\right|<\infty, 1 \leqslant i \leqslant t$, and $\bar{A}=\left\langle a_{1}, \ldots, a_{t}\right\rangle^{G}$ is a finite normal subgroup of $G$ contained in $A$. Consider the group $S \bar{A}$ generated by subnormal subgroups $(H \cap S) \bar{A}$ and $(K \cap S) \bar{A}$. Then $S \bar{A}$ satisfies the hypothesis of the theorem and the hypercentral residual is finite. By the above and [10], $(S \bar{A})^{*}=(H \cap S)^{*}(K \cap S)^{*} \leqslant B$. Moreover, $(S \bar{A})^{*}$ is normalized by $S$ and $A$ and so $(S \bar{A})^{*} \triangleleft G$. Thus $(S \bar{A})^{*}=1$ and $S \bar{A}$ is hypercentral. We deduce that $S A$ is locally nilpotent and therefore $G$ is hypercentral. The theorem is thus true in this case. 
Case 2. $B T<A$. Without loss of generality we may factor $G$ by $T$ to assume $A$ is torsion-free. Furthermore, by [6, Part 1, p. 157] we may assume that $N / A$ is free abelian-by-finite. Then there exists $V \leqslant N$ such that $A \leqslant V, V / A$ is torsion-free and $|G: V|<\infty$. Since $\mid G$ : $\operatorname{Core}_{G}(V) \mid<\infty$ we may assume $V / A \triangleleft G / A$. It follows then by [6, Part 2, Lemma 6.37] that $V / A \leqslant \zeta_{1}(G / A)=Z / A$. Hence $V \leqslant Z \leqslant N_{G}(B)$ and as in Case $1,\left|G: N_{G}(B)\right|<\infty$ and $G / A$ is nilpotent.

Let $U=\langle H \cap N, K \cap N\rangle$; then $U / B$ is generated by two normal hypercentral subgroups and hence is hypercentral. Since $\left|G: N_{G}(B)\right|<\infty, U /$ Core $_{G}(B)$ is hypercentral and since $B$ is core-free, $U$ is hypercentral. But $U$ is torsion-free and hence nilpotent by [6, Part 2, p. 38], and since $A=F(G), U \leqslant A$. Since $U \leqslant A$ then $H \cap N=A$ and $H / A$ is finite. Similarly $K / A$ is finite which implies $G / A$ is finite and $\left|G: C_{G}(X)\right|<\infty$. Thus $A$ satisfies the minimal condition and hence $A=1$ since $A$ is torsion free.

\section{REFERENCES}

1. M. Drukker, D. J. S. Robinson and I. N. Stewart, The subnormal coalescence of some classes of groups of finite rank, J. Austral. Math. Soc. 16 (1973), 324-327. MR 49 \#191

2. B. Hartley and M. J. Tomkinson, Splitting over nilpotent and hypercentral residuals, Math. Proc. Cambridge Philos. Soc. 78 (1975), 215-226.

3. A. G. Kurosh, The theory of groups, 2nd ed., 2 vols., Chelsea, New York, 1960. MR 22 \#27

4. J. C. Lennox, D. Segal and S. E. Stonehewer, The lower central series of a join of subnormal subgroups, Math. Z. 154 (1977), 85-89.

5. D. J. S. Robinson, Residual properties of some classes of infinite soluble groups, Proc. London Math. Soc. (3) 18 (1968), 495-520. MR 37 \#166

6. Finiteness conditions and generalized soluble groups, Parts 1 and 2, Springer-Verlag. Berlin/Heidelberg/New York, 1972. MR 48 \#11314 and \#11315

7. S. E. Stonehewer, The join of finitely many subnormal subgroups, Bull. London Math. Soc. 2 (1970), 77-82. MR 41 \#1877

8.

9. J. Whitehead, On certain properties of subnormal subgroups, Canad. J. Math. 30 (1978), 573-582.

10. H. Wielandt, Vertauschbare nachinvariante Untergruppen, Abh. Math. Sem. Univ. Hamburg 21 (1957), 55-62. MR $19 \# 387$

Department of Mathematics, NorthWestern University, EVAnSton, Illinois 60201

Current address: Department of Computer Science, Queens College, Flushing, New York 11367 